\title{
Integral Equations in Neutrino Mass Searches from Beta Decay
}

\author{
Thomas M. Semkow ${ }^{1}, \mathrm{Xin} \mathrm{Li}^{2}$ \\ ${ }^{1}$ Wadsworth Center, New York State Department of Health, Albany, NY, USA \\ ${ }^{2}$ School of Public Health, University at Albany, State University of New York, Rensselaer, NY, USA \\ Email: thomas.semkow@health.ny.gov
}

How to cite this paper: Semkow, T.M. and Li, X. (2019) Integral Equations in Neutrino Mass Searches from Beta Decay. Journal of Applied Mathematics and Physics, 7, 31-45.

https://doi.org/10.4236/jamp.2019.71004

Received: December 11, 2018

Accepted: January 7, 2019

Published: January 10, 2019

Copyright (c) 2019 by author(s) and Scientific Research Publishing Inc. This work is licensed under the Creative Commons Attribution International License (CC BY 4.0).

http://creativecommons.org/licenses/by/4.0/

(c) (i) Open Access

\begin{abstract}
A new mathematical model for elucidating neutrino mass from beta decay is proposed. It is based upon the solutions of transformed Fredholm and Abel integral equations. In principle, theoretical beta-particle spectra can consist of several neutrino-mass eigenstates. Integration of the beta spectrum with a normalized instrumental response function results in the Fredholm integral equation of the first kind. This equation is then transformed to yield a solution in a form of superposition of Heaviside step-functions, one for each neutrino mass eigenstate. A series expansion leading to matrix linear equations is then derived to solve the transformed Fredholm equation. Another approach is derived when the theoretical beta spectrum is obtained by a separate deconvolution of the observed spectrum. It is then proven that the transformed Fredholm equation reduces to the Abel integral equation. The Abel equation has a general integral solution, which is proven in this work by using a specific equation for the beta spectrum. Several examples of numerical solutions of the Abel equation are provided, which show a fractional sensitivity of about $10^{-3}$ for subtle neutrino eigenstate searches and can distinguish from the beta-spectrum discrepancies, such as minute shape and energy nonlinearities.
\end{abstract}

\section{Keywords}

Fredholm Equation, Abel Equation, Heaviside Function, Heavy Neutrino

\section{Introduction}

We start by summarizing the physics of neutrino-mass searches. Since the neutrino was conceptually proposed by Pauli in 1930 and given its name by Fermi, followed by his development of the beta decay theory in 1933-1934 [1] [2] [3] 
studies on this elusive neutral particle have never ceased. Whether or not neutrino bears a rest mass involves the extension of the Standard Model, the composition of Dark Matter in the Universe, and the asymmetry between matter and antimatter. According to the theory of neutrinos, mass eigenstates mix to result in the flavor eigenstates, the latter bear masses close to zero [4] [5]. In addition, a massive sterile neutrino has been sought [5] [6]. In this work, we will abbreviate neutrino "eigenstate" any measurable neutrino, which can have zero or positive mass, without referring to a specific generation mechanism.

One of the approaches to the neutrino mass searches has been studying an endpoint region of a beta-particle spectrum [7]. The Mainz and Troitsk experiments for ${ }^{3} \mathrm{H}$ beta decay yielded the upper limits of electron antineutrino mass at $2.3 \mathrm{eV}$ [8] and $2.05 \mathrm{eV}$ [9], respectively, while the KATRIN experiment is expected to have a sensitivity down to $0.2 \mathrm{eV}$ [10] [11].

In beta $\left(\beta^{-}\right)$decay, $\beta$-particle (electron) and electron antineutrino, $\bar{v}_{\mathrm{e}}$ (hereinafter referred to as neutrino) are emitted in the weak-interaction process of neutron decay inside the nucleus $\mathrm{n} \rightarrow \mathrm{p}^{+}+\beta^{-}+\bar{v}_{\mathrm{e}}$. The emitted $\beta$-particle kinetic-energy spectrum is a continuous function, owing to the energy sharing between the beta particle and the neutrino. Possible neutrino mass $M$ in the beta spectrum is included in the factor $\sqrt{(Q-T)^{2}-M^{2}}$, where $Q$ is the nuclear-recoil corrected $Q$-value of beta decay and $T$ is the emitted electron kinetic energy. The maximum beta energy (called the endpoint energy) is equal to $Q$ or $Q-M$, when the neutrino mass is zero or positive, respectively. In principle, there can be several mixed neutrino eigenstates. The details of the theoretical beta spectra are described in Section 2.

The emitted beta spectrum is convoluted with the instrumental response to yield the observed beta spectrum. The existing methods of identification of the neutrino mass in beta decay, whether close to or away from the endpoint energy, employ two approaches: 1) convolution of the theoretical beta spectrum with the normalized instrumental response function and comparison of the convolution with the observed spectrum, and 2) deconvolution of the observed spectrum and comparison with the theoretical spectrum [6]-[11]. Statistical measures, such as $\chi^{2}$ minimization or Bayesian likelihood, are used for the above-mentioned comparisons. Statistical measures can be non-specific i.e., there may be several factors other than neutrino mass, which affect them.

In order to advance beyond the difficulties and uncertainties associated with the statistical measures used for neutrino mass detection in beta decay of the existing methods, we aimed in this work at developing a novel mathematical theory based on transformational properties, rather than statistical measures. In Section 3, we derive a transformed Fredholm equation of the first kind, which includes both theoretical beta spectrum and instrumental response function, and show that the solution to it is a superposition of Heaviside step-functions with an abscissa of $(Q-T)^{2}$, one for each possible neutrino mass eigenstate. The neutrino mass can then be identified by the abscissa value at the raise of the step 
function, whereas its eigenstate contribution is shown to be proportional to the ordinate increment past the step. In Section 4, we outline a possible solution to the transformed Fredholm equation using series expansion and linear algebra.

If method 2) of deconvoluting the beta spectrum first is employed, before comparison with the theory, then the transformed Fredholm equation from Section 3 is shown to reduce to the Abel integral equation in Section 5, also known as the Abel transformation. The Abel integral equation, which is a special case of the Volterra integral equation of the first kind, has a known integral solution. We prove as a Lemma in Section 5 that, if the function undergoing Abel transformation has a form proportional to the beta spectrum, then the solution to the Abel equation is a Heaviside step-function. In Section 6, we provide numerical examples of solutions of the Abel equation, when the beta spectrum contains several neutrino-mass eigenstates. We also describe how the Abel solution behaves in the presence of small experimental discrepancies, which can influence neutrino mass detection, such as beta shape correction or small nonlinearities in the measured beta-energy scale. We summarize the advantages and limitations of the proposed new mathematical method of elucidating neutrino mass in beta decay in Section 7, as well as propose future directions of this work.

\section{Beta-Decay Theory}

There exist established formalisms for calculating allowed and superallowed beta spectra, which differ in some approaches [7] [12] [13]. For the purpose of this work, we define the beta-energy spectrum as follows:

$$
\dot{N}(T)=A D(T)(Q-T) \sqrt{(Q-T)^{2}-M^{2}},
$$

where $\dot{N}(T)=\mathrm{d} N / \mathrm{d} T$ represents a distribution of beta particles with energies between and $T$ and $T+\mathrm{d} T$, factor $A$ contains the quantum-transition matrix element and an overall normalization, whereas $Q, T$ and $M$ have been defined in Section 1.

Factor $D(T)$ in Equation (1) is defined as follows:

$$
D(T)=p E F_{n} F_{r} C_{r} S
$$

where $p$ is relativistic momentum, $E$ is total (relativistic) energy, $F_{n}$ is a non-relativistic Fermi function, $F_{r}$ is a relativistic correction to the Fermi function [14] and $C_{r}$ is a radiative correction [12]. $S$ is a shape correction [12], which corrects for small smooth discrepancies between the theoretical and observed spectra at low energies, and is assumed to be a parabola with an argument $Q-T$. Also, a screening correction [15] [16] is applied to $D(T)$. Additional term involving the progeny's final excitation states in low beta-energy emitters [7] as well as a possible Lorentz-invariance violation correction [17] have not been included.

The examples of beta spectra from ${ }^{3} \mathrm{H}$ decay $(Q=18590.29 \mathrm{eV})$, calculated according to Equations ((1), (2)) are plotted in Figure 1. The physical constants and nuclear parameters for the calculations were taken from [18]. It is seen that 


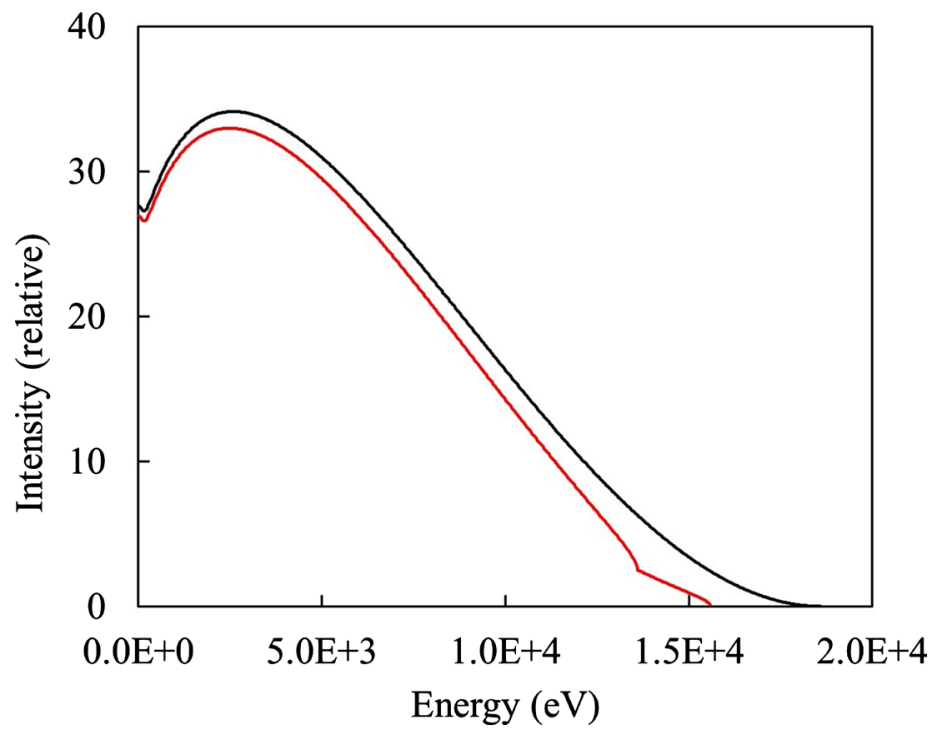

Figure 1. Beta particle spectra from ${ }^{3} \mathrm{H}$ decay. Black spectrum: zero neutrino mass; red spectrum: equal superposition of two neutrino eigenstates with the masses of 3000 and $5000 \mathrm{eV}$.

if neutrino mass is non-zero, the endpoint beta energy lies below $Q$, while another neutrino mass eigenstate is evidenced by the non-differentiable point in the spectrum. If neutrino mass is close to zero and any other eigenstate has a very small contribution, these effects are difficult to discern from the beta spectrum.

\section{Transformed Fredholm Integral Equation}

The observed beta spectrum usually differs from the emitted (theoretical) beta spectrum, given by Equations ((1), (2)), owing to an instrumental response. Let $T$ represent the emitted kinetic energy, while $T^{\prime}$ the observed kinetic energy. The emitted and observed beta spectra are then $\dot{N}(T)$ and $\dot{N}\left(T^{\prime}\right)$, respectively. They are coupled with each other by the response function $R\left(T^{\prime}, T\right)$ through the integral:

$$
\dot{N}\left(T^{\prime}\right)=\int_{0}^{Q-M} R\left(T^{\prime}, T\right) \dot{N}(T) \mathrm{d} T .
$$

Equation (3) is a Fredholm integral equation of the first kind [19], where $R\left(T^{\prime}, T\right)$ is a kernel. The variable $T^{\prime}$ can extend past $Q-M$ due to the abovementioned instrumental response. The response function is measured separately. Since the emission and detection of beta particles are stochastic processes, the normalized $R\left(T^{\prime}, T\right)$ and $\dot{N}(T)$ can be considered statistical probability density functions (pdf). The integral in Equation (3) is then a mixture of distributions [20]. If, additionally, $R\left(T^{\prime}, T\right)$ is a function of $T^{\prime}-T$ e.g., a Gaussian, then Equation (3) is a mathematical convolution [21]. The process of solving Equation (3) for $\dot{N}(T)$ is referred to as deconvolution and is discussed in Section 4. The terms: convolution and deconvolution will be used here even if Equation (3) is not a strictly mathematical convolution. 
If there existed $k$ neutrino-mass eigenstates with the masses $M_{k}$ and normalization factors $A_{k}$, then combining Equations ((1), (3)) and summing over the eigenstates would result in the observed spectrum:

$$
\dot{N}\left(T^{\prime}\right)=\sum_{k} A_{k} \int_{0}^{Q-M_{k}} R\left(T^{\prime}, T\right) D(T)(Q-T) \sqrt{(Q-T)^{2}-M_{k}^{2}} \mathrm{~d} T .
$$

Subsequently, we introduce a mass variable $m$ and utilize the Dirac $\delta$-function [22]. Then Equation (4) transforms to:

$\dot{N}\left(T^{\prime}\right)=\sum_{k} A_{k} \int_{0}^{Q} \mathrm{~d} m \delta\left(m-M_{k}\right) \int_{0}^{Q-m} \mathrm{~d} T R\left(T^{\prime}, T\right) D(T)(Q-T) \sqrt{(Q-T)^{2}-m^{2}}$.

New variables are defined: $x=\left(Q-T^{\prime}\right)^{2}$ and $z=(Q-T)^{2}$. By substitution, Equation (5) results in

$$
\dot{N}(x)=1 / 2 \sum_{k} A_{k} \int_{0}^{Q} \mathrm{~d} m \delta\left(m-M_{k}\right) \int_{m^{2}}^{Q^{2}} \mathrm{~d} z R(x, z) D(z) \sqrt{z-m^{2}} .
$$

By using a property of the $\delta$-function [22],

$$
\delta\left(m-M_{k}\right)=2 m \delta\left(m^{2}-M_{k}^{2}\right)
$$

and substituting of the variables, $y=m^{2}, a=Q^{2}$, and $a_{k}=M_{k}^{2}$, one obtains from Equation (6):

$$
\dot{N}(x)=1 / 2 \sum_{k} A_{k} \int_{0}^{a} \mathrm{~d} y \delta\left(y-a_{k}\right) \int_{y}^{a} \mathrm{~d} z R(x, z) D(z) \sqrt{z-y} .
$$

Changing the order of integration in Equation (8), yields

$$
\dot{N}(x)=1 / 2 \sum_{k} A_{k} \int_{0}^{a} \mathrm{~d} z R(x, z) D(z) \int_{0}^{z} \mathrm{~d} y \delta\left(y-a_{k}\right) \sqrt{z-y} .
$$

The inner integral in Equation (9) can be integrated by parts. We also use the fact that the integral of the $\delta$-function on positive argument is the Heaviside step function:

$$
\int \delta\left(y-a_{k}\right) \mathrm{d} y=H_{a_{k}}(y)= \begin{cases}0, & y \leq a_{k} \\ 1, & y>a_{k}\end{cases}
$$

The result is given below:

$$
\dot{N}(x)=1 / 4 \sum_{k} A_{k} \int_{0}^{a} \mathrm{~d} z R(x, z) D(z) \int_{0}^{z} \mathrm{~d} y H_{a_{k}}(y) / \sqrt{z-y} .
$$

The solution to Equation (11) is the function

$$
f(y)=\sum_{k} A_{k} H_{a_{k}}(y)
$$

which is a superposition of the step functions for all possible neutrino mass eigenstates, scaled by their appropriate contributions. By inserting Equation (12) into Equation (11) one obtains

$$
\dot{N}(x)=1 / 4 \int_{0}^{a} \mathrm{~d} z R(x, z) D(z) \int_{0}^{z} \mathrm{~d} y f(y) / \sqrt{z-y} .
$$

By reversing the order of integration in Equation (13) back to that of Equation (8) and by dividing both sides by $D(x)$ i.e., the theoretical factor $D$ given by Eq. (2) evaluated at the observed energy $T^{\prime}$, one obtains 


$$
\begin{aligned}
& \frac{\dot{N}(x)}{D(x)}=\int_{0}^{a} K(x, y) f(y) \mathrm{d} y, \text { with } \\
& K(x, y)=1 / 4 \int_{y}^{a} \frac{R(x, z)}{\sqrt{z-y}} \frac{D(z)}{D(x)} \mathrm{d} z .
\end{aligned}
$$

Equation (14a) is the transformed Fredholm equation. The kernel is given by Equation (14b). It links the observed beta spectrum with the emitted (theoretical) spectrum and with the instrumental response. In the solution function given by Equation (12), one does not need to assume the presence of neutrino mass or its eigenstates. They may be revealed by the abscissa and ordinate of any step functions present in the solution, to within the accuracy of the solution, which is dependent on the detailed shape of the observed spectrum. Therefore, the proposed approach interrogates the shape of the observed spectrum using the transformation derived, in contrast to the methods based on statistical measures.

\section{Solution of the Fredholm Integral Equation}

There exist established algorithms for solving Fredholm equations given by Equations (3) and (14a) [23] [24]. If Equation (3) was a mathematical convolution, then it could also be solved by the Laplace transform method, taking the advantage of the theorem that the Laplace transform of the convolution is a product of Laplace transforms of the convoluted functions [25].

However, Equation (14a) is not a convolution and we derive below a matrix method to solve it. We take into consideration the fact that the observed $T^{\prime}$ variable is discrete and incremented at equally spaced intervals (channels) owing to the instrumental digitizing of the spectrum (modern instrumentation can have $n=2^{15}$ or more channels). Consequently, variable $x$ is also digitized. Consider a general integral equation of the first kind, of which Equations ((3), (14a)) are the examples:

$$
\phi(x)=\int K(x, y) \psi(y) \mathrm{d} y .
$$

If $x$ is discrete, one has:

$$
\phi\left(x_{i}\right)=\int K\left(x_{i}, y\right) \psi(y) \mathrm{d} y, \quad i=1, \cdots, n .
$$

Let us assume a trial solution in a form of a series:

$$
\psi(y)=\sum_{j=1}^{n} K\left(x_{j}, y\right) c_{j} .
$$

By inserting the trial solution into Equation (16), one obtains a set of linear equations:

$$
\phi\left(x_{i}\right)=\sum_{j=1}^{n} K_{i j} c_{j}, \quad i=1, \cdots, n,
$$

with

$$
K_{i j}=\int K\left(x_{i}, y\right) K\left(x_{j}, y\right) \mathrm{d} y .
$$

In matrix notation, Equation (18a) reads: 


$$
\Phi=K C,
$$

where $\Phi$ and $\boldsymbol{C}$ are vectors and $\boldsymbol{K}$ is a symmetric square matrix. By solving the linear equations for $C$, the solution (17), continuous in $y$ variable (i.e., in $\left.(Q-T)^{2}\right)$ can, in principle, be obtained.

\section{Abel Integral Equation and Its Solution}

In Section 3, the general treatment for elucidating of neutrino mass in beta decay was presented, leading to the transformed Fredholm Equation (14a). This equation can be considerably simplified if the observed beta spectrum was first deconvoluted using Equation (3). The limit to the transformed Fredholm Equation (14a) can then be obtained by using the fact that, if the spectrum was deconvoluted, then for the purpose of Equation (14a),

$$
R\left(T^{\prime}, T\right)=\delta\left(T^{\prime}-T\right)=\delta\left[(Q-T)-\left(Q-T^{\prime}\right)\right]=\delta(\sqrt{z}-\sqrt{x})=2 \sqrt{z} \delta(z-x),
$$

where we used a version of Equation (7). Then, starting from Equation (13),

$$
\begin{aligned}
\dot{N}(x) & =1 / 4 \int_{0}^{a} \mathrm{~d} z \delta(z-x) 2 \sqrt{z} D(z) \int_{0}^{z} \mathrm{~d} y f(y) / \sqrt{z-y} \\
& =1 / 2 D(x) \sqrt{x} \int_{0}^{x} f(y) / \sqrt{x-y} \mathrm{~d} y .
\end{aligned}
$$

By defining a new function:

$$
g(x)=\frac{\dot{N}(x)}{D(x) \sqrt{x}}
$$

we obtain from Equation (21)

$$
g(x)=\frac{1}{2} \int_{0}^{x} \frac{f(y)}{\sqrt{x-y}} \mathrm{~d} y .
$$

Equation (23) is a special case of the Abel transformation, also called the Abel integral equation [19] [26], which is a special case of the Volterra integral equation of the first kind. The general form of the Abel equation is

$$
g(x)=\int_{0}^{x}(x-y)^{-\alpha} f(y) \mathrm{d} y, \quad 0<\alpha<1,
$$

with a solution of

$$
f(x)=\frac{\sin \pi \alpha}{\pi} \frac{\mathrm{d}}{\mathrm{d} x} \int_{0}^{x}(x-y)^{\alpha-1} g(y) \mathrm{d} y .
$$

Therefore, the solution of Equation (23) for $\alpha=1 / 2$ is

$$
f(x)=\frac{2}{\pi} \frac{\mathrm{d}}{\mathrm{d} x} \int_{0}^{x} \frac{g(y)}{\sqrt{x-y}} \mathrm{~d} y .
$$

By using Equation (1) for a single neutrino-mass eigenstate $M$, and setting $y=(Q-T)^{2}$ as well as $b=M^{2}$, we obtain from Equation (22)

$$
g(y)=A \sqrt{y-b} .
$$

Lemma. If $g(y)$ is given by Equation (27), then $f(x)=A H_{b}(x)$, where $H$ 
is the Heaviside step function given by Equation (10).

Proof. By inserting Equation (27) to Equation (26), we obtain

$$
\begin{gathered}
f(x)= \begin{cases}0, & y \leq b \\
\frac{2 A}{\pi} \frac{\mathrm{d}}{\mathrm{d} x} \int_{b}^{x} \frac{\sqrt{y-b}}{\sqrt{x-y}} \mathrm{~d} y, & x>y>b .\end{cases} \\
\int_{b}^{x} \frac{\sqrt{y-b}}{\sqrt{x-y}} \mathrm{~d} y \stackrel{\text { by parts }}{\longrightarrow} \frac{1}{2}(x-b) \int_{b}^{x} \frac{\mathrm{d} y}{\sqrt{(y-b)(x-y)}}=\frac{\pi}{2}(x-b),
\end{gathered}
$$

since the last integral is equal to $\pi$ [27]. Whence,

$$
\begin{aligned}
f(x) & = \begin{cases}0, & x \leq b \\
A \frac{\mathrm{d}}{\mathrm{d} x}(x-b)=A, & x>b\end{cases} \\
& =A H_{b}(x),
\end{aligned}
$$

which proves the Lemma.

If there are $k$ neutrino mass eigenstates $M_{k}, a_{k}=M_{k}^{2}$, with relative contributions $A_{k}$, then the function $g$ from Equation (27) can be written as:

$$
g(y)=\sum_{k} A_{k} \sqrt{y-a_{k}},
$$

and, according to the Lemma, generalization of the solution to the Abel equation is given by Equation (12).

\section{Numerical Examples}

In this Section, several numerical calculations are provided for the solution to the Abel equation. In a possible application of this method to the observed spectrum, function $g$ in Equation (22) is defined as the ratio of the deconvoluted beta-energy spectrum $\dot{N}(T)$, obtained from Equation (3), to calculated $D(T)(Q-T)$ using Equation (2). Since we do not know a priori how many neutrino eigenstates there are and what are their corresponding masses, one needs to perform a numerical solution on function $g$ using Equation (26). However, for the purpose of this calculation, we assumed one or more eigenstate components in a simulated spectrum $\dot{N}(T)$, calculated according to Equation (1), without deconvolution. For numerical integration and differentiation of the test function using Equation (26), we applied the established high-performance algorithms [23] with an additional adaptive optimization of the differential step.

In the first calculation, we demonstrate how the step-function solution $f$ depends on the bin size of abscissa $x$, for mass-less neutrino. Several numerical solutions for zero neutrino-mass ${ }^{3} \mathrm{H}$ decay are plotted in Figure 2 as functions of the $x=(Q-T)^{2}$ argument for different bin sizes. The leftmost points at $x=0$ could not be plotted on a logarithmic scale, so they were placed on the abscissa at the smallest bin studied, $x=0.01 \mathrm{eV}^{2}$. Therefore, the raise of the step for a given bin size can be taken as an upper limit of the square of neutrino mass.

In the second calculation, we selected beta decay of ${ }^{35} \mathrm{~S}(Q=167.319 \mathrm{keV}[18])$ 


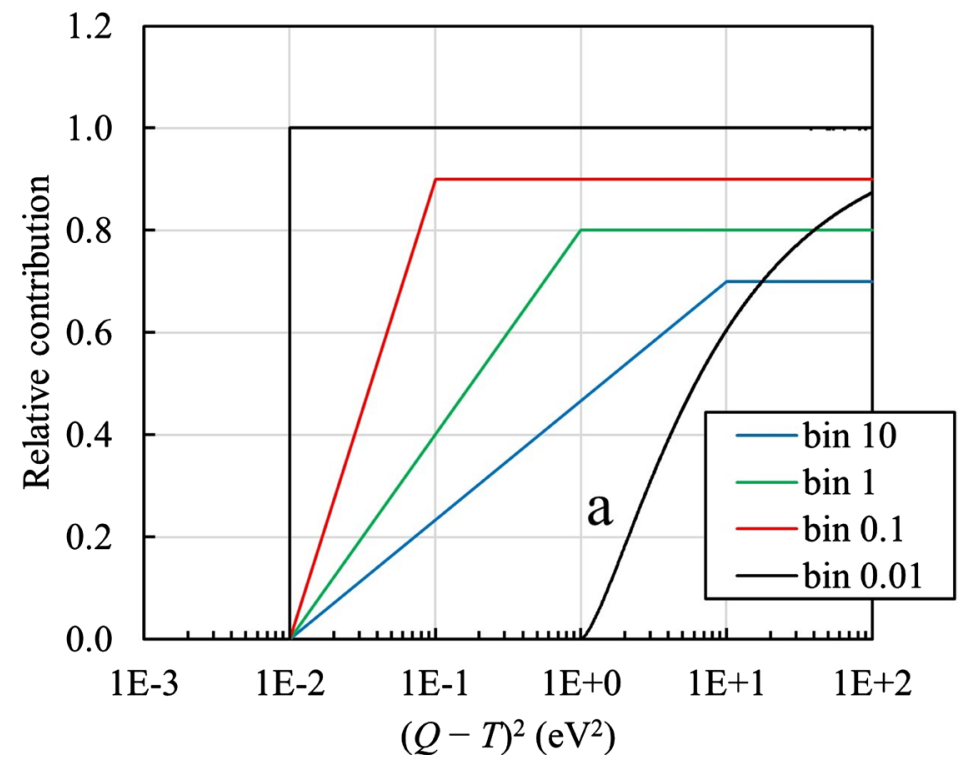

Figure 2. Numerical solutions of the Abel equation for zero neutrino mass ${ }^{3} \mathrm{H}$ decay (except curve (a)), plotted for various abscissa bin sizes in $\mathrm{eV}^{2}$ indicated in the legend. Zero-abscissa values are arbitrarily placed at $0.01 \mathrm{eV}^{2}$. The relative contributions are scaled to $1.0,0.9,0.8$, and 0.7 to enable better visualization. Curve (a) depicts the effect of energy nonlinearity for $0.01 \mathrm{eV}^{2}$ bin.

Table 1. Assumed and identified parameters for 3-neutrino mass eigenstate test of the numerical solution of the Abel equation for searching of neutrino mass in beta decay of ${ }^{35} \mathrm{~S}$.

\begin{tabular}{cccc}
\hline \multirow{2}{*}{ Parameter } & \multicolumn{3}{c}{ Eigenstate } \\
\cline { 2 - 4 } & 1 & 2 & 3 \\
\hline Assumed neutrino mass $(\mathrm{keV})$ & 0 & 10 & 15 \\
Assumed contribution & 1 & 0.001 & 0.001 \\
Identified contribution & $1.000 \mathrm{E}+00$ & $9.997 \mathrm{E}-04$ & $1.000 \mathrm{E}-03$ \\
Relative uncertainty & $2.2 \mathrm{E}-11$ & $1.2 \mathrm{E}-02$ & $1.7 \mathrm{E}-02$ \\
\hline
\end{tabular}

and assumed 3 neutrino eigenstates with the masses of 0,10 , and $15 \mathrm{keV}$ as well as their contributions (normalization factors) of $1,0.001$, and 0.001 , respectively, as given in Table 1.

Function $g$ was calculated using Equation (31), and subjected to the numerical solution using Equation (26). The solution $f$ is plotted as a function of argument $(Q-T)^{2}$ in Figure 3 (black curve), emphasizing the vicinity of 1 on the ordinate axis. It is seen that the three step functions are easily identified. The raise of the step functions on the abscissa of the numerical solution correctly identify squares of the neutrino masses at 0,100 , and $225 \mathrm{keV}$, respectively. The identified contributions given in Table 1 agree with the assumed contributions. While the method is in principle non-statistical, the relative uncertainties of the identified contributions are caused by rounding errors in the numerical solution. The relative uncertainty is negligible for the principal eigenstate and between $1 \%$ $2 \%$ for the minor eigenstates. 
When comparing experimental and theoretical beta spectra, small smooth discrepancies were observed, which increase with decreasing kinetic energy [12]. They can be corrected for with a shape-correction factor $S$, which is assumed to be a parabola with an argument $Q-T$. An example of such a shape correction function is given in Figure 4 (black curve) for ${ }^{35} \mathrm{~S}$ decay. This correction factor is equal to one at a maximum kinetic energy, and it is assumed 1.01 at zero energy. In the following, we analyzed the effect of this correction on the Abel solution. We assumed that $\dot{N}(T)$ had a deviation from a theoretical shape according to the shape correction function in Figure 4, included in $D(T)$ in Equation (2), and did not correct for it when dividing by $D(T)$ in Equation (22). We also

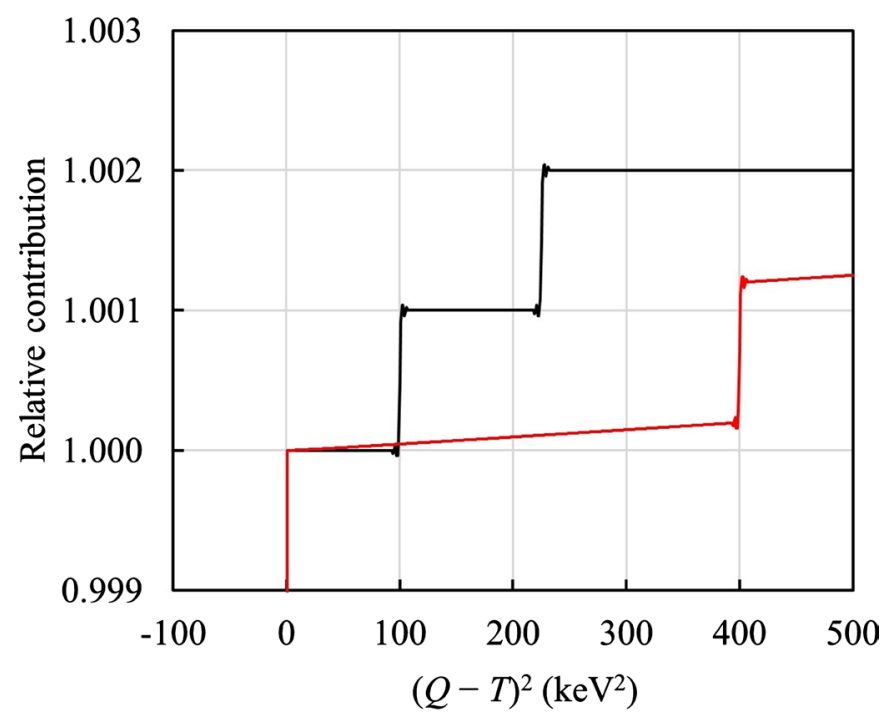

Figure 3. Numerical solution of the Abel equation for searching of neutrino mass in the beta decay of ${ }^{35} \mathrm{~S}$. Black curve: three neutrino components with masses of 0,10 , and 15 $\mathrm{keV}$. Red curve: two neutrino components with masses 0 and $20 \mathrm{keV}$ with a shape correction function.

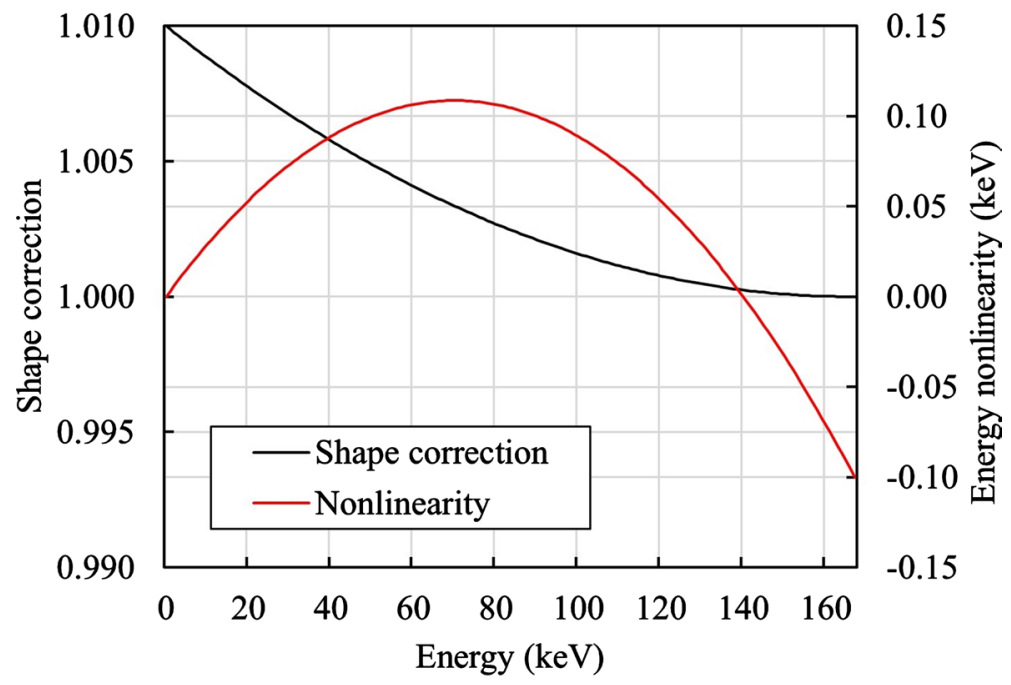

Figure 4. Shape correction and energy nonlinearity plotted as functions of energy for ${ }^{35} \mathrm{~S}$ decay. 
assumed two neutrino mass eigenstates at 0 (contribution of 1) and $20 \mathrm{keV}$ (contribution of 0.001). Subsequent application of the Abel solution resulted in a red curve in Figure 3. It is seen that not correcting for shape results in the flat portions of the step function sloping upward, however, both the neutrino masses and their contributions were unaffected.

Besides the shape correction, which affects the intensity of the beta spectrum (in the ordinate direction), we study the effect of energy-scale nonlinearities (in the abscissa direction) on the Abel solution and neutrino-mass detection for ${ }^{35} \mathrm{~S}$ decay. Such minute nonlinearities may result from improper energy calibration or from nonlinear response of a digital signal processor. As a next example, we assumed the energy nonlinearity with a functional dependence as depicted in Figure 4 (red curve). We calculated $\dot{N}(T)$ with the energy nonlinearities included and did not correct for them in $D(T)$ for Equation (21). We study the effects close to the maximum kinetic energy (endpoint energy, at zero neutrino mass) and at lower kinetic energies (for a possible presence of heavy neutrino) separately.

For any nonlinearity that is positive at the end-point energy and is not corrected for, the deconvoluted energy would exceed $Q$ and thus would be identified as an experimental problem. Therefore, we assumed nonlinearity equal to $-0.1 \mathrm{keV}$ at the maximum kinetic energy (see Figure 4). Such nonlinearity would result in the observed energy being shifted down by $0.1 \mathrm{keV}(\sim 0.06 \%)$ below $Q$. This might simulate the presence of neutrino mass. The Abel solution for this case is depicted in Figure 5. Without nonlinearities present (black curve), the upper limit on the square of neutrino mass would be placed at $0.001 \mathrm{keV}^{2}$. The above-mentioned nonlinearity appear to simulate a square of the neutrino mass at $0.01 \mathrm{keV}^{2}$ (red curve), however, it can be rejected because the step is not sharp but is a rather slowly raising function instead.

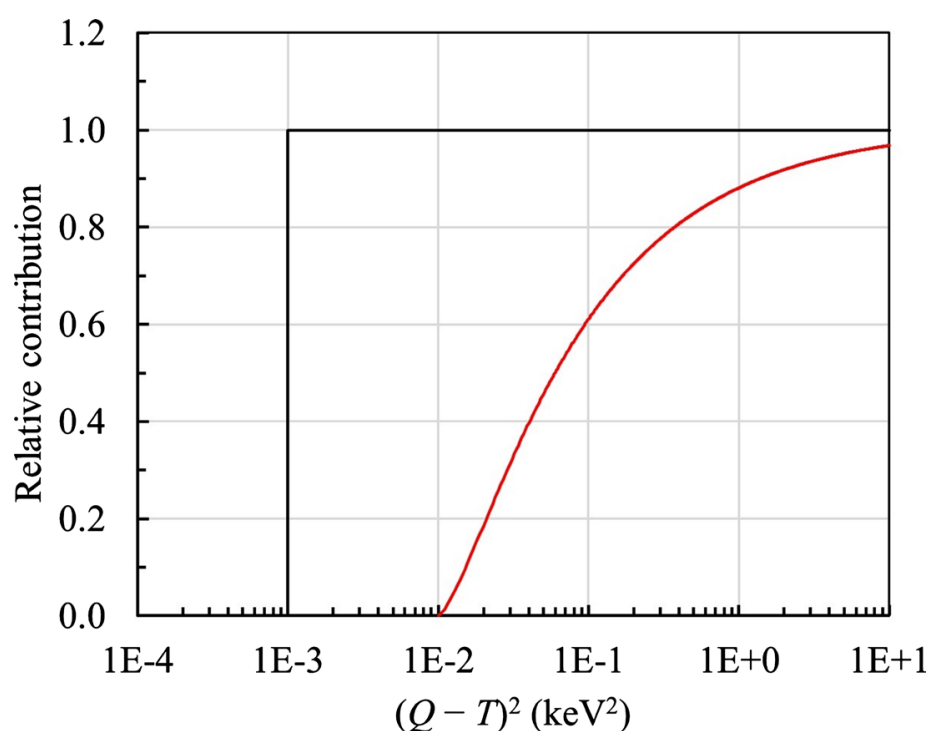

Figure 5. Step function solutions for zero neutrino mass ${ }^{35} \mathrm{~S}$ decay, with (red curve) and without (black curve) energy nonlinearities. 


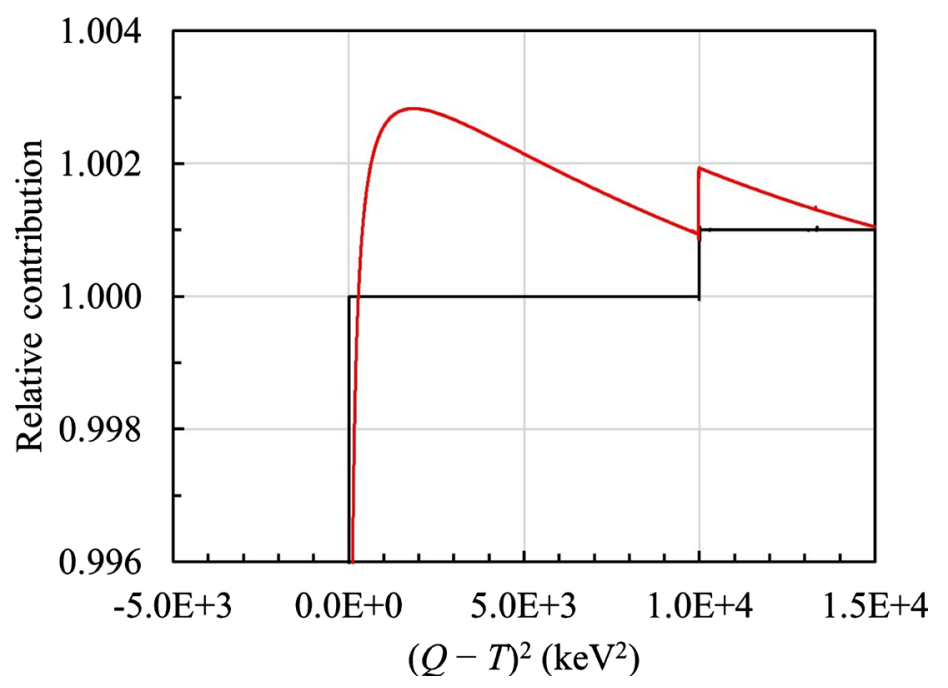

Figure 6. Step function solutions for ${ }^{35} \mathrm{~S}$ decay consisting of two neutrino components having mases of 0 and $100 \mathrm{keV}$, with (red curve) and without (black curve) energy nonlinearities.

The effect of the energy nonlinearity from Figure 4 on the wider range of the Abel solution is depicted in Figure 6 (red curve), where we have also added a $100-\mathrm{keV}$ neutrino component at 0.001 contribution. It is seen that the curve resembles an exact step at $10^{4} \mathrm{eV}^{2}$, as it does in the case without nonlinearities depicted by the black curve. Therefore the heavy neutrino is identified at $100 \mathrm{keV}$ and only its mass is shifted by the $0.1 \mathrm{keV}$ nonlinearity (see Figure 4).

We have also assessed the effect of energy nonlinearities on neutrino mass in ${ }^{3} \mathrm{H}$ decay. We assumed a functional nonlinearity similar to the one in Figure 4, except the energy shift at the endpoint was assumed to be $-1 \mathrm{eV}(\sim 0.005 \%)$ below $Q$. The Abel solution resulted in curve (a) in Figure 2. It appears to simulate a square of the neutrino mass at $1 \mathrm{eV}^{2}$, however, it can be rejected following the arguments for ${ }^{35} \mathrm{~S}$ in Figure 5.

\section{Summary and Conclusions}

We have developed a math-theoretical model for searches of neutrino mass in beta-decay energy spectra. The transformed Fredholm equation was derived (Equation (14a) with the kernel given by Equation (14b)), linking the observed beta spectrum with the theoretical one as well as with the instrumental response. The solution to this equation is a superposition of the Heaviside step-functions representing the possible neutrino mass eigenstates, as well as their contributions (Equation (12)). In this way, the shape of beta spectrum is tested for nondifferentiable points, which could be associated with the neutrino mass. The step function is a very characteristic signature of such non-differentiabilities. A possible solution to the Fredholm equation was derived based on a series expansion (Equation (17)) and solution of the matrix equation (Equation (19)), taking advantage of the fact that the observed spectrum is discrete. Numerical verification of the transformed Fredholm equation deserves further investigation. 
A limiting version of the transformed Fredholm equation was also derived. It is based upon prior normal deconvolution of the observed spectrum from the instrumental response using Equation (3). The factors dependent on neutrino mass (Equation (31)) are then subjected to the Abel transformation (Abel integral equation, Equation (23)), the solution to which (Equation (26)) is proven in the Lemma to be a superposition of the step functions. The Abel transformation was numerically tested on theoretically calculated beta spectra. It was shown that this method is sensitive down to a fractional contribution of about $10^{-3}$ for heavy neutrinos and can set upper limits on the neutrino mass of $0.1 \mathrm{eV}$ for ${ }^{3} \mathrm{H}$ and $0.03 \mathrm{keV}$ for ${ }^{35} \mathrm{~S}$.

We have also numerically tested two possible experimental deviations in beta spectra: shape correction and energy nonlinearities. It was shown that a smooth, moderate shape correction up to $1 \%$ at low energy changes the slope of the step function but does not affect the numerical recognition of either zero-mass or heavy neutrino. Likewise, heavy neutrino detection is not affected by energy nonlinearity. However, the energy nonlinearity can simulate an artificial neutrino mass close to the endpoint of beta spectrum. Nevertheless, if the Abel method is applied to such a case, the step function is not sharp and, therefore, the hypothesis of neutrino mass can be rejected. If not neutrino mass, the proposed method can indicate some discrepancies between observed and calculated beta spectra.

The novelty of the model presented in this work is that the solutions with the step functions are very sensitive and characteristic signatures of neutrino mass, and they can in many cases distinguish between experimental discrepancies and true neutrino-mass signatures. Therefore, this model may have some advantages over the statistical methods, which may be less specific and less sensitive for neutrino mass detection in beta decay.

The math-theoretical methods derived here are inherently non-statistical, however they have possible limitations arising from numerical errors, theoretical considerations, and statistical fluctuations present in the observed spectra. They are discussed below in that order.

It was shown that at the $10^{-3}$ level, the Abel-transformation method resulted in relative fluctuations below $2 \%$, originating from rounding errors in the calculations. Such rounding errors could be possibly lowered, resulting in a higher sensitivity, if higher-precision arithmetic were used in the numerical calculations.

The theory of beta decay with all possible corrections, from which theoretical beta spectra are derived, can be given only to a finite accuracy, which is a limiting factor for neutrino mass searches in beta decay, regardless of the method used [12].

Finally, the statistical fluctuations in the observed beta spectra limit the sensitivity of neutrino-mass searches in beta decay by any method, including this one. Owing to the fact that the observed spectrum is discrete, there are Poisson fluctuations in each spectrum channel. The variation coefficient of the Poisson fluc- 
tuations is given by $1 / \sqrt{N}$, where $N$ is the number of observed counts in the channel [21]. As seen in Figure 1, the fluctuations are expected to be lower at the low beta energy region and higher close to the endpoint. The fluctuations present in the observed spectrum will translate into the fluctuations of any solution derived from the spectrum. It is, therefore, imperative to have the observed statistics in the data as high as possible. One way to alleviate problems with low statistics could be fitting of a polynomial to such data and applying integral equations to the polynomial fit instead of original data points.

\section{Acknowledgements}

Thanks are due to C. J. Bradt for his valuable comments.

\section{Conflicts of Interest}

The authors declare no conflicts of interest regarding the publication of this paper.

\section{References}

[1] Fermi, E. (1933) Tentativo di Una Teoria dell'Emissione dei Raggi "beta". Ric. Scientifica, 4, 491-495.

[2] Fermi, E. (1934) Versuch Einer Theorie der $\beta$-Strahlen. Zeitschrift für Physik, 88, 161-171. https://doi.org/10.1007/BF01351864

[3] Wilson, F.L. (1968) Fermi's Theory of Beta Decay. American Journal of Physics, 36, 1150-1160. https://doi.org/10.1119/1.1974382

[4] Giunti, C. and Kim, C.W. (2007) Fundamentals of Neutrino Physics and Astrophysics. Oxford University Press, Oxford. https://doi.org/10.1093/acprof:oso/9780198508717.001.0001

[5] Bilenky, S. (2018) Introduction to the Physics of Massive and Mixed Neutrinos. Springer, Berlin. https://doi.org/10.1007/978-3-319-74802-3

[6] Chang, S. (2016) Sterile Neutrinos Give IceCube and Other Experiments the Cold Shoulder. Physics Today, 69, 15-19. https://doi.org/10.1063/PT.3.3316

[7] Drexlin, G., Hannen, V., Mertens, S. and Weinheimer, C. (2013) Current Direct Neutrino Mass Experiments. Advances in High Energy Physics, 2013, Article ID: 293986. https://doi.org/10.1155/2013/293986

[8] Kraus, C., Bornschein, B., Bornschein, L., et al. (2005) Final Results from Phase II of the Mainz Neutrino Mass Search in Tritium $\beta$ Decay. The European Physical Journal C, 40, 447-468. https://doi.org/10.1140/epjc/s2005-02139-7

[9] Aseev, V.N., Belesev, A.I., Berlev, A.I., et al. (2011) Upper Limit on the Electron Antineutrino Mass from the Troitsk Experiment. Physical Review D, 84, 112003. https://doi.org/10.1103/PhysRevD.84.112003

[10] Mertens, S. (2016) Direct Neutrino Mass Experiments. Journal of Physics: Conference Series, 718, 022013. https://doi.org/10.1088/1742-6596/718/2/022013

[11] Feder, T. (2017) Massive Machine Gears up to Weigh Nearly Massless Particles. Physics Today, 70, 26-29. https://doi.org/10.1063/PT.3.3656

[12] Bahran, M., Chen, W.R. and Kalbfleisch, G.R. (1993) Fermi Theory of Nuclear Decay and Heavy Neutrino Searches. Physical Review D, 47, R759-R763. https://doi.org/10.1103/PhysRevD.47.R759 
[13] Mougeot, X. (2016) Systematic Comparison of Beta Spectra Calculations Using Improved Analytical Screening Correction with Experimental Shape Factors. Applied Radiation and Isotopes, 109, 177-182. https://doi.org/10.1016/j.apradiso.2015.11.030

[14] Behrens, H. and Jänecke, J. (1969) Numerical Tables for Beta-Decay and Electron Capture. In: Hellwege, K.-H., Ed., Landolt-Börnstein Numerical Data and Functional Relationships in Science and Technology, Springer-Verlag, Berlin.

[15] Rose, M.E. (1936) A Note on the Possible Effect of Screening in the Theory of Beta-Disintegration. Physical Review, 49, 727-729. https://doi.org/10.1103/PhysRev.49.727

[16] Durand, L. (1964) Electron Screening Corrections to Beta-Decay Spectra. Physical Review, 135, B310-B313. https://doi.org/10.1103/PhysRev.135.B310

[17] Carmona, J.M. and Cortés, J.L. (2000) Testing Lorentz Invariance Violations in the Tritium Beta-Decay Anomaly. Physics Letters B, 494, 75-80. https://doi.org/10.1016/S0370-2693(00)01182-5

[18] Huang, W.J., Audi, G., Wang, M., Kondev, F.G., Naimi, S. and Xu, X. (2017) The Ame2016 Atomic Mass Evaluation, (I). Evaluation of Input Data; and Adjustment Procedures. Chinese Physics C, 41, Article ID: 030002.

[19] Porter, D. and Stirling, D.S.G. (1993) Integral Equations. Cambridge University Press, Cambridge, Sections 1.2, 1.3.4, 9.2.

[20] Johnson, N.L., Kotz, S. and Balakrishnan, N. (1994) Continuous Univariate Distributions, Vol. 1. Wiley-Interscience Publication, New York, Section 12.3.

[21] Johnson, N.L., Kemp, A.W. and Kotz, S. (2005) Univariate Discrete Distributions. Wiley-Interscience, Hoboken, Sections 1.2.11, 4.4. https://doi.org/10.1002/0471715816

[22] Dirac, P.A.M. (1988) The Principles of Quantum Mechanics. Oxford University Press, Oxford, Section III.15.

[23] Numerical Algorithms Group Ltd., Oxford, UK.

[24] Wolfram Research, Champaign, IL, USA.

[25] Margenau, H. and Murphy, G.M. (1976) The Mathematics of Physics and Chemistry. Krieger, Huntington, Section 8.5.

[26] Titchmarsh, E.C. (1967) Introduction to the Theory of Fourier Integrals. Oxford University Press, Oxford, Section 11.14.

[27] Gradshteyn, I.S. and Ryzhik, I.M. (2015) Table of Integrals, Series, and Products. 8th Edition, Academic Press, Boston, Section 2.61. 